\title{
Erratum to: Determination of the Johnson-Cook Constitutive Model Parameters of Materials by Cluster Global Optimization Algorithm
}

\author{
Zhipeng Huang, Lihong Gao, Yangwei Wang, and Fuchi Wang
}

\section{Erratum to: J. of Materi Eng and Perform \\ DOI 10.1007/s11665-016-2178-1}

Please note that all correspondence for this article should be sent to Lihong Gao (e-mail: gaolihong@bit.edu.cn).

The online version of the original article can be found under doi: 10.1007/s11665-016-2178-1.

Zhipeng Huang, School of Materials Science and Engineering, Beijing Institute of Technology, Beijing 100081, China; and Lihong Gao, Yangwei Wang, and Fuchi Wang, School of Materials Science and Engineering, Beijing Institute of Technology, Beijing 100081, China; and National Key Laboratory of Science and Technology on Materials under Shock and Impact, Beijing 100081, China. Contact e-mails: hzp1560@126.com and gaolihong@bit.edu.cn. 